decrease in the glomerular filtration rate would constitute clinical tumor lysis syndrome (see Table 1 of our article). Removal of a $25 \%$ change from the definition by Cairo and Bishop applies only to uric acid, phosphorus, potassium, and calcium. The lack of randomized studies precludes definitive recommendation of a specific diuretic. For now, the rat model provides the only available data, and furosemide has proved effective in our practice.

Scott C. Howard, M.D.

St. Jude Children's Research Hospital

Memphis, TN

scott.howard@stjude.org

Deborah P. Jones, M.D.

Vanderbilt University School of Medicine Nashville, TN
Ching-Hon Pui, M.D.

St. Jude Children's Research Hospital Memphis, TN

Since publication of their article, the authors report no further potential conflict of interest.

1. Clark TG, Fry AE, Auburn S, et al. Allelic heterogeneity of G6PD deficiency in West Africa and severe malaria susceptibility. Eur J Hum Genet 2009;17:1080-5.

2. Giraldez M, Puto K. A single, fixed dose of rasburicase $(6 \mathrm{mg}$ maximum) for treatment of tumor lysis syndrome in adults. Eur J Haematol 2010;85:177-9.

3. Knoebel R, Lo M, Crank C. Evaluation of a low, weight-based dose of rasburicase in adult patients for the treatment or prophylaxis of tumor lysis syndrome. J Oncol Pharm Pract 2010 March 23 (Epub ahead of print).

4. Maheshwari A, Misro MM, Aggarwal A, Sharma RK, Nandan D. N-acetyl-L-cysteine counteracts oxidative stress and prevents $\mathrm{H} 2 \mathrm{O} 2$ induced germ cell apoptosis through down-regulation of caspase-9 and JNK/c-Jun. Mol Reprod Dev 2011;78:69-79.

\title{
Fiberoptic Intubation
}

TO THE EDITOR: In his video on fiberoptic intubation (May 19 issue), ${ }^{1}$ Heidegger provides excellent points for patients with a known or suspected difficult airway. Some readers may infer that there is a single way of preparing the patient and performing a fiberoptic intubation, yet multiple methods may be used for these procedures. The sizes of the endotracheal tube and the fiberoptic scope can vary according to several clinical factors (e.g., a smaller tube for suspected airway edema or subglottic narrowing). Also, I would suggest that it is preferable to have the patient in a sitting position with the laryngoscopist facing the patient, since this position optimizes airway anatomy. To prepare the patient, local anesthetics other than cocaine (e.g., lidocaine and tetracaine) are routinely used, and other vasoconstrictors (e.g., phenylephrine and oxymetazoline) can minimize the risk of intranasal bleeding. The administration of intravenous fentanyl (at a dose of $2 \mu \mathrm{g}$ per kilogram of body weight) may be hazardous to some patients, and sedation often is not required or not safe. The intratracheal injection of local anesthetic is not necessary in many patients and, in those with a tenuous airway, could result in violent coughing and loss of the airway.

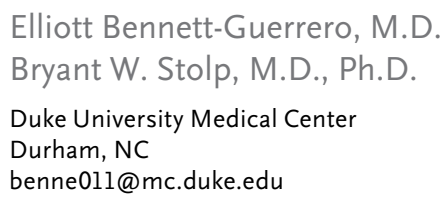

No potential conflict of interest relevant to this letter was reported.

1. Heidegger T. Fiberoptic intubation. N Engl J Med 2011;364(20): e42. (Available at NEJM.org.)

TO THE EDITOR: In patients who are undergoing fiberoptic intubation, we believe that it is essential to distinguish between awake and asleep techniques. The indications for an awake procedure, as presented by Heidegger, are too broad and, as described, might put the patient in unnecessary danger. Poor dentition, a beard, or obesity are not common indications for awake fiberoptic intubation and reflect what we consider to be an outdated approach to the management of a difficult airway. According to our practice, awake fiberoptic intubation is appropriate only when the patient is at risk for being in a "cannot intubate, cannot ventilate" situation (e.g., in cases of stridor secondary to airway cancer or neck mass). ${ }^{1}$ The primary objective is to maintain patency of the upper airway until it is secured by the orotracheal tube. To avoid oversedation and upper-airway obstruction, any long-acting drug, such as clonidine or fentanyl, which may produce an alteration of consciousness, should not be used, especially not in combination. In our judgment, an ultrashort-acting opioid, such as remifentanil, which overcomes this problem, is preferable. $^{2}$ 
Eric Albrecht, M.D.

Patrick Schoettker, M.D.

Centre Hospitalier Universitaire Vaudois

Lausanne, Switzerland

eric.albrecht74@gmail.com

No potential conflict of interest relevant to this letter was reported.

1. Practice guidelines for management of the difficult airway: an updated report by the American Society of Anesthesiologists Task Force on Management of the Difficult Airway. Anesthesiology 2003;98:1269-77. [Erratum, Anesthesiology 2004;101:565.]

2. Puchner W, Egger P, Pühringer F, Löckinger A, Obwegeser J, Gombotz H. Evaluation of remifentanil as single drug for awake fiberoptic intubation. Acta Anaesthesiol Scand 2002;46:350-4.

TO THE EDITOR: The video on fiberoptic intubation provides a thorough illustration and narrative of the fiberoptic-intubation technique. However, we feel that certain issues bear discussion. The author recommends oxygen insufflation through the bronchoscope during intubation to assist with atomization of local anesthetic. This practice is unnecessary and should be avoided in small children and patients with airway edema, since the egress of gas around the bronchoscope may be impaired, thereby increasing the risk of pneumothorax. ${ }^{1}$

The administration of a hypnotic before advancement of the tracheal tube is suggested as a method for performing fiberoptic intubation without patient discomfort. In our experience, most patients have no discomfort with trachealtube placement after effective topical anesthesia. Furthermore, the rationale for performing fiberoptic intubation while the patient is awake is usually based on concern about difficulty with ventilation. If a hypnotic is administered and tracheal-tube placement is difficult (e.g., the tube hangs up at the glottis or esophageal intubation occurs), a situation may arise in which the airway is unsecured and ventilation is impossible.

John Fiadjoe, M.D.

Paul Stricker, M.D.

University of Pennsylvania

Philadelphia, PA

fiadjoej@email.chop.edu

No potential conflict of interest relevant to this letter was reported.

1. Khan RM, Sharma PK, Kaul N. Barotrauma: a life-threatening complication of fiberoptic endotracheal intubation in a neonate. Paediatr Anaesth 2010;20:782-4.
THE AUTHOR REPLIES: As stated in the video and in the accompanying supplementary text (and as correctly addressed by Bennett-Guerrero and Stolp), there are different ways to prepare the patient and perform fiberoptic intubation. The choice of equipment is primarily dependent on personal preference and local circumstance. Whether fiberoptic intubation should be performed in the sitting position is also primarily dependent on the physician who performs the procedure. However, from my experience, it is very important to perform the procedure as you normally would, especially in difficult situations. The combination of oral clonidine and $2 \mu \mathrm{g}$ per kilogram of intravenous fentanyl normally has only a mild and desired sedative effect. However, as mentioned in the article, no sedating medication should be given to patients with a severely compromised airway, not even during insertion of the fiberscope.

Reducing the indication of awake fiberoptic intubation to patients who are at risk for a "cannot intubate, cannot ventilate" situation, as recommended by Albrecht and Schoettker, is potentially dangerous and against common sense and established practice ("Daily practice is the key to success in emergencies"). It is well known that the common appearance of more risk factors is a strong predictor of difficult mask ventilation. ${ }^{1,2}$ A recent survey that was based on data from nearly 2.9 million patients who underwent general anesthesia in the United Kingdom showed that obese patients are at increased risk for an obstructed airway because of difficult mask ventilation, difficult intubation, or both. ${ }^{3}$ In an editorial on the survey results, the authors concluded that "obesity must now be regarded as a significant material risk in airway management" and called for the development of evidence-based algorithms for airway management in patients who were at high risk for difficult intubation. ${ }^{4}$ I am convinced that fiberoptic intubation will play an important part in such cases.

The comments by Fiadjoe and Stricker regarding oxygen insufflation in small children are helpful. Continuous oxygen should be applied very carefully in adults as well. It is particularly dangerous if the bronchoscope is unintentionally advanced into the esophagus, which might lead to rupture of the stomach because of overinsufflation. 
In daily practice, the concept of fiberoptic intubation in the awake patient is not clearly defined. In most cases, the choice of technique is dependent on institutional and personal preferences. Ultimately, such a choice is a compromise between safety, practicability, and acceptance. The technique as shown in the video is a thoroughly documented, well-tested method that has not been changed for many years. ${ }^{5}$

Thomas Heidegger, M.D.

Spitalregion Rheintal Werdenberg Sarganserland Walenstadt, Switzerland

thomas.heidegger@srrws.ch
Since publication of his article, the author reports no further potential conflict of interest.

1. Langeron O, Masso E, Huraux C, et al. Prediction of difficult mask ventilation. Anesthesiology 2000;92:1229-36.

2. Kheterpal S, Martin L, Shanks AM, Tremper KK. Prediction and outcomes of impossible mask ventilation: a review of 50,000 anesthetics. Anesthesiology 2009;110:891-7.

3. Cook TM, Woodall N, Frerk C. Major complications of airway management in the UK: results of the Fourth National Audit Project of the Royal College of Anaesthetists and the Difficult Airway Society. Part 1: anaesthesia. Br J Anaesth 2011;106:617-31. 4. O'Sullivan E, Laffey J, Pandit JJ. A rude awakening after our fourth 'NAP': lessons for airway management. Anaesthesia 2011;66:331-4.

5. Heidegger T, Gerig HJ, Ulrich B, Schnider TW. Structure and process quality illustrated by fibreoptic intubation: analysis of 1612 cases. Anaesthesia 2003;58:734-9.

\section{Deferoxamine for Advanced Hepatocellular Carcinoma}

TO THE EDITOR: We have previously reported that the iron chelator deferoxamine can prevent liver injury as well as the development of preneoplastic lesions in rats, ${ }^{1,2}$ and we have proposed the use of deferoxamine as an anticancer drug. The antiproliferative effect of deferoxamine arrests the cell cycle and induces apoptosis. ${ }^{3}$ To our knowledge, no clinical study has been performed to evaluate deferoxamine therapy in patients with hepatocellular carcinoma. ${ }^{4}$

Our study involved 10 patients $(6$ men and 4 women) who had advanced hepatocellular carcinoma and did not have a response to hepatic arterial infusion chemotherapy with anticancer drugs. The average age of the patients was 64 years (range, 43 to 77). Written informed consent was obtained before the study, which was approved by the institutional review board of Yamaguchi University Hospital. Seven patients had hepatitis $C$ virus infection, 2 patients had hepatitis B virus infection, and 1 patient did not have either type of infection. The tumor stages were classified as II, IVA, and IVB (according to the Liver Cancer Study Group of Japan criteria) for 1, 2, and 7 patients, respectively. The Child-Pugh class was $\mathrm{A}, \mathrm{B}$, and $\mathrm{C}$ for 3,5 , and 2 patients, respectively. (In the Child-Pugh classification of liver disease, class A indicates the least severe disease, class B moderately severe disease, and class $\mathrm{C}$ the most severe disease.) The patients received an arterial infusion of deferoxamine (at a dose of 10 to 80 mg per kilogram of body weight) over 24 hours on alternate days, through the injection port.

Deferoxamine was administered an average of 27 times (range, 9 to 78). Two, three, and five patients had a partial response, stable disease, and progressive disease, respectively (according to the Eastern Cooperative Oncology Group criteria). The overall response rate was $20 \%$.

Tumor-marker levels (alpha-fetoprotein, des- $\gamma$ carboxyprothrombin, alpha-fetoprotein L3, or all of these levels) decreased in patients with a partial response. In one patient, a massive hepatocellular tumor with lung metastases disappeared with deferoxamine treatment (Fig. 1). The 1-year cumulative survival rate was $20 \%$. Four patients had grade 2 or 3 interstitial pneumonia (according to the Common Terminology Criteria for Adverse Events, version 4.0), and one patient had grade 2 renal dysfunction. However, no grade 4 adverse events were observed.

Sorafenib, a multikinase inhibitor, has recently been established as the standard of care for patients with advanced hepatocellular carcinoma and preserved liver function (Child-Pugh class A) because it increases survival. ${ }^{5}$ However, its safety and efficacy for patients with Child-Pugh class $\mathrm{B}$ or C disease is still unknown. Deferoxamine may warrant testing in patients with Child-Pugh class B or $\mathrm{C}$ hepatocellular carcinoma. 